\title{
ARTICLE OPEN \\ A dichotomy between model responses of tropical ascent and descent to surface warming
}

\author{
Hui Su ${ }^{1}{ }^{1}$, Chengxing Zhai ${ }^{1}$, Jonathan H. Jiang ${ }^{1}$, Longtao $\mathrm{Wu}^{1}$, J. David Neelin ${ }^{2}$ and Yuk L. Yung ${ }^{3}$
}

\begin{abstract}
Simulations of tropical atmospheric circulation response to surface warming vary substantially across models, causing large uncertainties in projections of regional precipitation change. Understanding the physical processes that drive the model spread in tropical circulation changes is critically needed. Here we employ the basic mass balance and energetic constraints on tropical circulation to identify the dominant factors that determine multidecadal circulation strength and area changes in climate models. We show that the models produce a robust weakening of descent rate under warming regardless of surface warming patterns; however, ascent rate change exhibits inter-model spread twice as large as descent rate because of diverse model responses in the radiative effects of clouds, water vapor, and aerosols. As ascent area change is dictated by the disparate descent and ascent rate changes due to the mass budget and the inter-model spread in descent rate change is small, the model spread in ascent area change is dominated by that of ascent rate change, resulting in a strong anti-correlation of -0.85 between the fractional changes of ascent strength and area across 77 climate model simulations. This anti-correlation leads to a corresponding inverse relationship between the rates of precipitation intensifying and narrowing of the inter-tropical convergence zone (ITCZ), suggesting tropical ascent area change can be potentially used to constrain the ITCZ precipitation change. Longwave cloud radiative effect at the topof-atmosphere (TOA) in the convective region is identified to be a major source of uncertainties for tropical ascent rate change and thus for regional precipitation change.
\end{abstract}

npj Climate and Atmospheric Science (2019)2:8 ; https://doi.org/10.1038/s41612-019-0066-8

\section{INTRODUCTION}

Accurate prediction of regional precipitation response to longterm surface warming is critical to decision making regarding adaptation and mitigation strategies. However, climate model predictions of regional precipitation change contain large uncertainties. Previous studies have shown that the circulationrelated dynamic component of precipitation change is the primary contributor to the inter-model variance in regional precipitation predictions. ${ }^{1,2}$ Thus, it is a top priority in climate research to better understand and reduce discrepancies in model simulations of atmospheric circulation response to surface warming. ${ }^{2}$

Tropical circulation systems such as the Hadley Circulation and Walker Circulation consist of prevailing ascending and descending regions. Thermodynamic theories postulate that the strength of tropical overturning circulation would weaken in response to surface warming in terms of both mean ascent and descent rates $^{3-5}$ and the ascent area would tighten ${ }^{6-9}$ while the descent area expands poleward. ${ }^{10,11}$ During the satellite era since 1979, many studies showed tropical circulation especially the Walker Circulation have strengthened along with a steady increase of global-mean surface temperature $\left(T_{s}\right)^{12-18}$ although a weakening of the Walker Cell was found in other studies that covered longer temporal periods, ${ }^{19,20}$ motivating a number of studies to reconcile the apparent contradiction between the thermodynamic theories and the observations.

Climate models driven by observed sea surface temperature (SST) approximately capture the Walker Circulation strengthening, but most coupled model simulations fail to reproduce the observed signal and a large inter-model spread exists in the simulated decadal circulation change. ${ }^{21-23}$ It was shown that the change of east-west SST gradient associated with the decadal variability in the tropical Pacific or the frequency of central-Pacific El Niño is responsible for the observed strengthening of the Walker Circulation since 1979. ${ }^{18,21-23}$ However, large differences exist in the simulated trends of the circulation strength even when observed SST is prescribed in the model experiments, demonstrating that model representations of atmospheric processes have significant discrepancies.

In this study, we present a unique perspective to decipher the diverse circulation responses to surface warming in climate models. Using a large number of simulations available from the Couple Model Inter-comparison Project Phase 5 (CMIP5), we seek robust and consistent signals across the models and identify the dominant physical processes that govern the inter-model spread in tropical circulation changes. In particular, we treat the tropics $\left(30^{\circ} \mathrm{S}-30^{\circ} \mathrm{N}\right)$ as two boxes corresponding to the ascending and descending branches of the overturning circulation with uniform ascent rate $\left(W_{u}\right)$ and descent rate $\left(W_{d}\right)$ in each box, respectively. ${ }^{24,25}$ The area fractions of the ascending and descending boxes are denoted as $A_{u}$ and $A_{d}$, respectively, with $A_{u}+A_{d}=1$. The caveats for this simplified framework are discussed later. We show that a weakening of $W_{d}$ with warming is simulated in all models with relatively small model spread, but $W_{u}$ can increase or decrease because of model differences in simulating the radiative

\footnotetext{
'Jet Propulsion Laboratory, California Institute of Technology, Pasadena, CA 91109, USA; ${ }^{2}$ Department of Atmospheric and Oceanic Sciences, University of California, Los Angeles, Los Angeles, CA 90095, USA and ${ }^{3}$ Division of Geological and Planetary Sciences, California Institute of Technology, Pasadena, CA 91125, USA

Correspondence: Hui Su (Hui.Su@jpl.nasa.gov)
}

Received: 13 October 2018 Accepted: 14 February 2019

Published online: 06 March 2019 
effects of clouds, water vapor, and aerosols. A strong anticorrelation exists between the fractional changes of $W_{u}$ and $A_{u}$ across the models because of the dichotomy between the model representations of the descent and ascent responses to warming. This anti-correlation was reported in Byrne et al. ${ }^{26}$ but its mechanism was not fully explored. We further show this anticorrelation leads to "the wetter the narrower" ITCZ response across the models. It is found that different longwave cloud radiative effects in the convective region are a primary contributor to the diverse $W_{u}$ and $A_{u}$ sensitivities to surface warming.

\section{RESULTS}

Ascent area change constrained by mass balance

The mass balance for the tropical circulation requires $W_{u} A_{u}=$ $W_{d} A_{d}$. In climatological means, the imbalance between the ascent mass flux $W_{u} A_{u}$ and the descent mass flux $W_{d} A_{d}$ within $30^{\circ} \mathrm{S}-30^{\circ} \mathrm{N}$ is only $2-3 \%$ (Supplementary Figure 1 ). As the Hadley Circulation expands poleward under global warming, ${ }^{10,11}$ the imbalance between the ascent and descent mass fluxes within $30^{\circ} \mathrm{S} / \mathrm{N}$ would increase. Let us first ignore the mass imbalance for simplicity but quantify its impact later. Making use of the relation $\frac{d A_{u}}{d T_{s}}=-\frac{d A_{d}}{d T_{s}}$ in the fixed latitudinal band, we can write the fractional change of the ascent area as

$\frac{1}{A_{u}} \frac{d A_{u}}{d T_{s}}=\left(\frac{1}{W_{d}} \frac{d W_{d}}{d T_{s}}-\frac{1}{W_{u}} \frac{d W_{u}}{d T_{s}}\right)\left(1-A_{u}\right)$.

Defining the ascending region by monthly-mean vertical pressure velocity at $500 \mathrm{hPa}\left(\omega_{500}\right)<0$, we compute the fractional changes of $W_{u}, W_{d}$ and $A_{u}$ per degree of $T_{s}$ increase using the linear trends of $W_{u}, W_{d}$, and $A_{u}$ scaled by their multi-year means and the global-mean $T_{s}$ trends from 1979 to 2005 in 28 atmosphere-only (AMIP) and 24 historical experiments and from 2006 to 2100 in 25 RCP4.5 experiments. The long-term $T_{s}$ trends are similar in the three types of experiments, all around $0.2 \mathrm{~K}$ decade $^{-1}$. The typical value of $A_{u}$ is about 0.4 in the models (Supplementary Figure 2), so $\left(1-A_{u}\right)=0.6$ is used when evaluating Eq (1) by scattering $\frac{1}{A_{u}} \frac{d A_{u}}{d T_{s}}$ against $\left(\frac{1}{W_{u}} \frac{d W_{u}}{d T_{s}}-\frac{1}{W_{d}} \frac{d W_{d}}{d T_{s}}\right)\left(1-A_{u}\right)$ for all experiments (Fig. 1).

Figure 1 shows that the mass balance places a dominant constraint on the ascent area change in the models. The diagnostic relation (Eq. 1) captures about $86 \%$ of the acrossmodel variance in $\frac{1}{A_{u}} \frac{d A_{u}}{d T_{s}}$, which confirms the validity of the simple two-box model in representing the mass balance of the threedimensional tropical circulation. It reveals that the ascent area change compensates for the differential responses of the descent and ascent rate to surface warming: The correlation between $\frac{1}{A_{u}} \frac{d A_{u}}{d T_{s}}$ and the r.h.s. of Eq (1) is 0.93 and all the experiments follow closely the 1:1 line. High correlations are found within each type of experiments ( 0.90 for AMIP, 0.89 for historical and 0.87 for RCP4.5 experiments). The deviation from the $1: 1$ line could be caused by the model spread in the exact values of $A_{u}$, the full width of the Hadley Circulation, and the poleward expansion of the Hadley Circulation with warming (see Supplementary Discussion, Supplementary Figures 2 and 3). As there is only $14 \%$ of the inter-model variance in $\frac{1}{A_{u}} \frac{d A_{u}}{d T_{S}}$ not explained by Eq (1), we use this simple framework to interpret the relationships between the changes in the circulation strength and area.

It is also evident in Fig. 1 that most (66 out of 77 ) model simulations produce a tightening of the ascent area under warming and the values of $\frac{1}{A_{u}} \frac{d A_{u}}{d T_{s}}$ range from $-6 \% \mathrm{~K}^{-1}$ to $2 \% \mathrm{~K}^{-1}$. The RCP4.5 runs generally have a small inter-model spread because a longer period is used in deriving the trends. Using a subset of 25 years of data from the RCP4.5 experiments yields larger spread, comparable to those in the AMIP and historical runs.

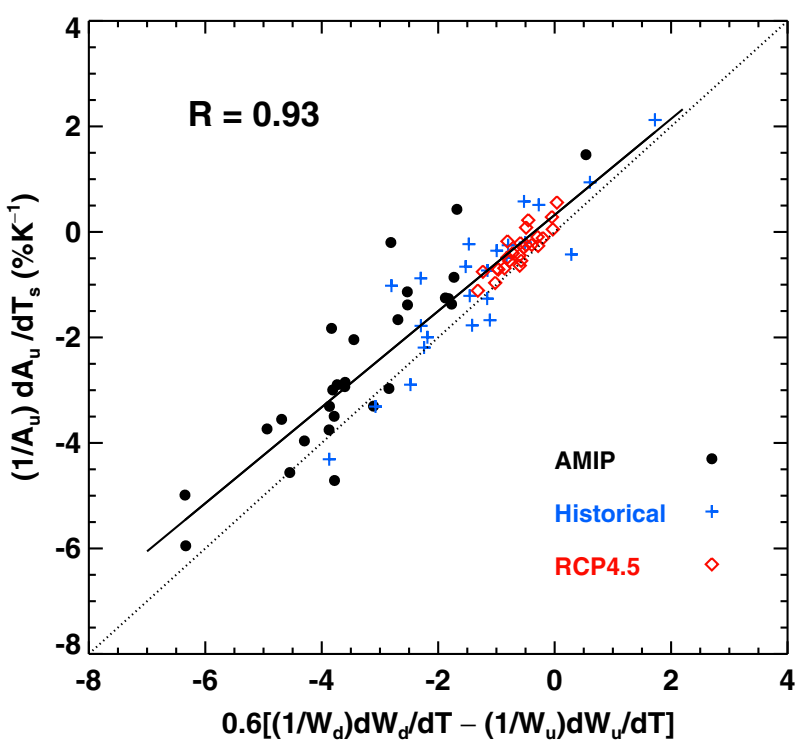

Fig. 1 Tropical ascent area change dictated by differential changes of descent and ascent rates. The fractional change of tropical ascent area scattered against the differential responses of mean descent rate and ascent rate to surface warming across the model simulations (see Eq (1)). All fractional changes are normalized by global-mean surface temperature trends over the same period. Each symbol represents a model experiment. The black line marks the least squares linear fit across all model experiments with correlation coefficient $(R)$ shown. The dotted line corresponds to $y=x$

The multi-model-mean $\frac{1}{A_{u}} \frac{d A_{u}}{d T_{s}}$ in the AMIP experiments is $-2.5 \% \mathrm{~K}^{-1}$, while the multi-mean-mean $\frac{1}{A_{u}} \frac{d A_{u}}{d T_{s}}$ in the historical and RCP4.5 experiments are $-1.0 \% \mathrm{~K}^{-1}$ and $-0.3 \% \mathrm{~K}^{-1}$, respectively. To understand the model behaviors in the $A_{u}$ change, we examine the physical processes that govern the changes of $W_{u}$ and $W_{d}$ and the dominant factors that are responsible for the model differences.

\section{Energetic control of the ascent and descent rates}

The moist static energy (MSE) budget framework combines thermodynamic and moisture equations and effectively cancels convective heating and moisture sink. It has been used extensively in studies of tropical dynamics. ${ }^{27-29}$ Considering the weak temperature and moisture gradients in the tropics and the dominance of the first baroclinic mode in vertical motion, ${ }^{30-32}$ the magnitude of $W_{u}$ is approximately determined by the net energy flux into the atmospheric column $\left(F_{\text {net }}^{u}\right)$ divided by gross moist stability (GMS), $W_{u}=F_{\text {net }}^{u} / \mathrm{GMS}$.

The GMS represents the efficiency of energy export out of the convective region given unit vertical ascent and is sensitive to the vertical structure of MSE and vertical motion. ${ }^{31,32}$ In this study, we analyze the relationship between $\frac{1}{F_{\text {net }}^{u}} \frac{d F_{\text {net }}^{u}}{d T_{s}}$ and $\frac{1}{W_{u}} \frac{d W_{u}}{d T_{s}}$ across the models, and the contributions of the GMS changes and other processes can be inferred from the residue, i.e.,

$\frac{1}{W_{u}} \frac{d W_{u}}{d T_{s}}=\frac{1}{F_{\text {net }}^{u}} \frac{d F_{\text {net }}^{u}}{d T_{s}}-\frac{1}{\mathrm{GMS}} \frac{d \mathrm{GMS}}{d T_{s}}$.

Similarly, over the tropical descent zone, the net energy loss in the free troposphere $\left(F_{\text {net }}^{d}\right)$ must be balanced by adiabatic warming associated with subsidence so that $W_{d}=F_{\text {net }}^{d} / \mathrm{DSS}$, where DSS represents the dry static stability. ${ }^{4}$ We use DSS $=-\frac{T}{\theta} \frac{\partial \theta}{\partial P}$ at $500 \mathrm{hPa}$, where $T$ is temperature and $\theta$ is potential temperature (see methods). Therefore, the fractional change of $W_{d}$ depends on the 


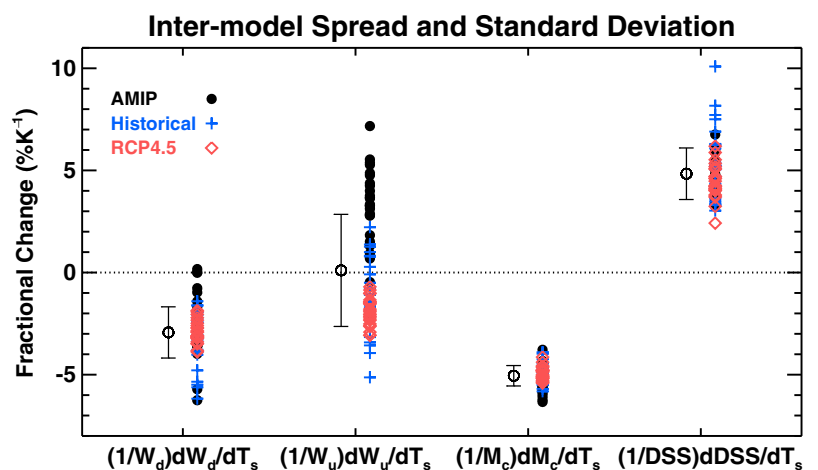

Fig. 2 Contrasting behaviors of the simulated trends in the descent and ascent rates in CMIP5 models. Inter-model spread and standard deviation in the fractional changes of mean descent rate $\left(W_{d}\right)$, mean ascent rate $\left(W_{u}\right)$, global-mean convective mass flux $\left(M_{c}\right)$, and dry static stability (DSS) per unit surface warming. Each symbol represents a model experiment. The open circle denotes the ensemble mean of all model experiments and the error bar denotes one inter-model standard deviation

fractional changes of $F_{\text {net }}^{d}$ and DSS, i.e.,

$\frac{1}{W_{d}} \frac{d W_{d}}{d T_{s}}=\frac{1}{F_{n e t}^{d}} \frac{d F_{n e t}^{d}}{d T_{s}}-\frac{1}{\mathrm{DSS}} \frac{d \mathrm{DSS}}{d T_{s}}$.

The diverse responses of $W_{d}$ and $W_{u}$ in the models are shown in Fig. 2. It is striking that all 77 simulations produce a weakening of $W_{d}$ despite of a variety of $T_{s}$ warming patterns, while the fractional change of $W_{u}$ per unit surface warming $\left(\frac{1}{W_{u}} \frac{d W_{u}}{d T_{s}}\right)$ varies from $-5 \%$ $\mathrm{K}^{-1}$ to $7 \% \mathrm{~K}^{-1}$. The inter-model standard deviation for $\frac{1}{W_{d}} \frac{d W_{d}}{d T_{s}}$ is only $1.3 \% \mathrm{~K}^{-1}$, compared to $2.7 \% \mathrm{~K}^{-1}$ for $\frac{1}{W_{u}} \frac{d W_{u}}{d T_{s}}$. Most AMIP runs (25 out of 28) produce a strengthening of $W_{u}$, but most historical runs (17 out 24) simulate a weakening of $W_{u}$. As shown in previous studies, the discrepancy between the AMIP and historical runs are mainly caused by the misrepresentation of SST gradient in the coupled simulations. ${ }^{18,21-23}$ All RCP4.5 experiments predict a weakening of $W_{u}$ and $W_{d}$ with relatively small inter-model spread comparable to their $W_{d}$ changes, consistent with the small values of $\frac{1}{A_{u}} \frac{d A_{u}}{d T_{s}}$ in the RCP4.5 runs (Fig. 1). Albern et al. (2018) ${ }^{33}$ showed that uniform SST warming leads to a robust weakening of descent rate in 8 aquaplanet model simulations regardless of whether or not cloudradiative interactions are included, but the ascent rate and area changes are more variable with even different signs, suggesting that the dichotomy between the descent and ascent responses to warming also occurs under idealized model configurations.

It is worth noting that the changes of $W_{u}$ per degree of surface warming do not follow the effective convective mass flux $\left(M_{c}\right)$ derived from the differential responses of global-mean precipitation and boundary layer moisture, $\frac{1}{M_{c}} \frac{d M_{c}}{d T_{s}}=\frac{1}{P} \frac{d P}{d T_{s}}-\frac{1}{q_{v}} \frac{d q_{v}}{d T_{s}}$, as suggested by early studies. ${ }^{3,5}$ The fractional changes of $M_{c}$ are universally negative between $-6.3 \% \mathrm{~K}^{-1}$ to $-3.8 \% \mathrm{~K}^{-1}$ with an ensemble mean of $-5.1 \% \mathrm{~K}^{-1}$ and one standard deviation of $0.5 \%$ $\mathrm{K}^{-1}$ (Fig. 2), and they do not have a simple relation with the gridscale $W_{u}$ changes (their correlation is 0.026 , Supplementary Figure 4a). The positive correlation between the changes of $M_{c}$ and $W_{u}$ shown in Fig. 4 of Vecchi and Soden ${ }^{5}$ originates from the covariability of both quantities with the $T_{s}$ increase (Supplementary Figure $4 \mathrm{~b})$. Given unit surface warming, $W_{u}$ can increase or decrease, but $M_{c}$ always decreases. It is important to distinguish these two quantities even though in some cases their changes are of the same sign, such as for centennial changes in the RCP4.5 runs (Fig. 2).
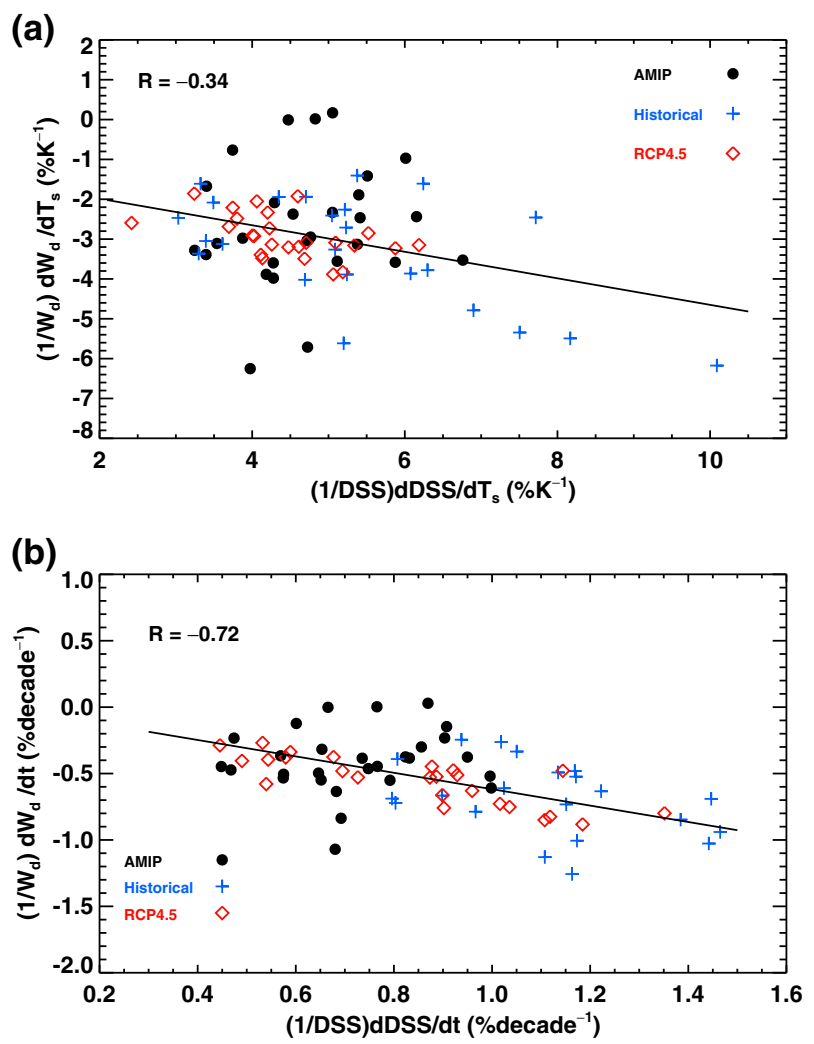

Fig. 3 Weakening of descent rate governed by the increase of dry static stability with warming. The fractional change of mean descent rate scattered against the fractional change of dry static stability averaged over the tropical descending region (a) with and (b) without normalization by global-mean surface temperature trend for the same period. Each symbol represents a model experiment. The black line marks the least squares linear fit across all model experiments with correlation coefficient $(R)$ shown

In contrast, the weakening of $W_{d}$ in all models is consistent with the expectation that the increase of DSS with warmer $T_{s}$ dominates over the increase of radiative cooling. ${ }^{4,34}$ When the surface warms, tropical tropospheric temperature profiles tend to follow moist adiabats so that upper troposphere warms more than lower troposphere, resulting in a more stable atmosphere. The increase of DSS with warming is consistently simulated in all models, despite of quantitative differences between the experiments (Fig. 2). The ensemble-mean $\frac{1}{\mathrm{DSS}} \frac{d \mathrm{DSS}}{d T_{s}}$ for all experiments is about $4.8 \% \mathrm{~K}^{-1}$ and the standard deviation is $1.3 \% \mathrm{~K}^{-1}$, close to that of $\frac{1}{W_{d}} \frac{d W_{d}}{d T_{s}}$.

A scatterplot between the simulated $\frac{1}{W_{d}} \frac{d W_{d}}{d T_{s}}$ against the corresponding $\frac{1}{\mathrm{DSS}} \frac{d \mathrm{DSS}}{d T_{s}}$ (Fig. 3a) reveals a negative correlation of -0.34 (statistically significant at $99 \%$ level), while the fractional change of $W_{d}$ without normalization of the $T_{s}$ trend yields a higher negative correlation of -0.72 with that of DSS (Fig. $3 b$ ), confirming the dominance of the DSS change in driving the reduction of large-scale subsidence. The correlation between $\frac{1}{W_{d}} \frac{d W_{d}}{d T_{s}}$ and $\frac{1}{\mathrm{DSS} S} \frac{d \mathrm{DSS}}{d T_{s}}$ is 0.13 (statistically insignificant) for AMIP, -0.60 for historical and -0.57 for RCP4.5. The insignificant correlation in the AMIP experiments may be caused by the relatively small inter-model variance of $\frac{1}{\mathrm{DSS}} \frac{d \mathrm{DSS}}{d T_{s}}$ associated with the same prescribed SSTs, which does not overcome the considerable noises in the shortterm trends of DSS. On the other hand, the signal-to-noise ratios are much higher in the historical and RCP4.5 experiments (the uncertainties of the long-term trends are much smaller than the uncertainties of the short-term trends) so that the negative 
(a)

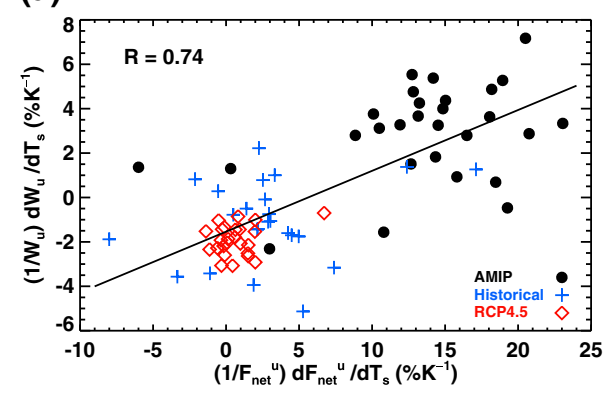

(b)

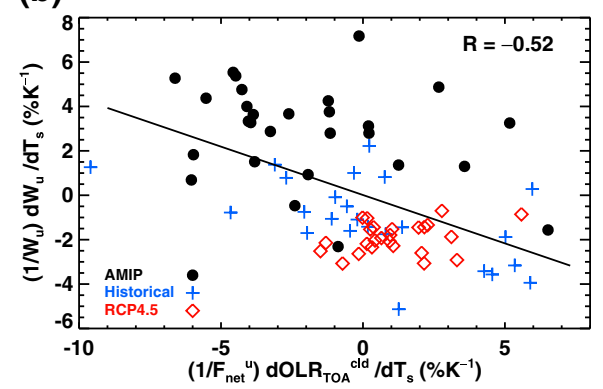

(c)

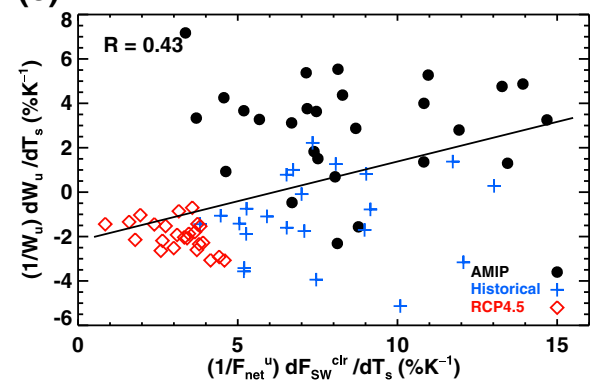

Fig. 4 Ascent rate change driven by net energy flux into the atmospheric column. The fractional change of mean ascent rate scattered against a the fractional change of net energy flux into the atmospheric column, $\mathbf{b}$ the longwave component of cloud radiative effect at the top-of-atmosphere, and c the clear-sky shortwave absorption in the atmosphere averaged over the tropical ascending region across the model simulations. All fractional changes are normalized by global-mean surface temperature trends over the same period. Each symbol represents a model experiment. The black line marks the least squares linear fit across all model experiments with correlation coefficient $(R)$ shown

correlations between $\frac{1}{W_{d}} \frac{d W_{d}}{d T_{s}}$ and $\frac{1}{\mathrm{DSS}} \frac{d \mathrm{DSS}}{d T_{s}}$ are outstanding. Without normalization of the $T_{s}$ trend, the correlation between $\frac{1}{W_{d}} \frac{d W_{d}}{d t}$ and $\frac{1}{\mathrm{DSS}} \frac{d \mathrm{DSS}}{d t}$ is -0.77 for the historical runs and -0.81 for the RCP4.5 runs, but insignificant for the AMIP runs.

The relatively small inter-model spread in the $T_{s}$-normalized $\frac{1}{W_{d}} \frac{d W_{d}}{d T_{s}}$ and $\frac{1}{\mathrm{DSS}} \frac{d \mathrm{DSS}}{d T_{s}}$ suggests that the sensitivities of $W_{d}$ and DSS per degree of surface warming are fairly consistent among the models. Model differences in convective adjustment and radiative effects of water vapor and clouds could cause different tropospheric temperature changes and result in different DSS sensitivities and thus $W_{d}$ changes, but their inter-model spreads are about half of that in $\frac{1}{W_{u}} \frac{d W_{u}}{d T_{s}}$ (Fig. 2). On the other hand, the model spreads in $\frac{1}{W_{d}} \frac{d W_{d}}{d T_{s}}$ and $\frac{1}{F_{\text {net }}^{d}} \frac{d F_{\text {net }}^{d}}{d T_{s}}$ are not significantly correlated (figure not shown).
Different from $W_{d}$, the model spread in $\frac{1}{W_{u}} \frac{d W_{u}}{d T_{s}}$ is significantly influenced by the change of net energy flux into the atmospheric column (Fig. 4a). The AMIP experiments generally have greater net energy flux increase, contributing to a strengthening of ascent, while the historical runs have lower net energy flux input and reduced ascent. The RCP4.5 runs are clustered together with nearly neutral $F_{\text {net }}^{u}$ change. We recognize that the significant correlation of 0.74 between $\frac{1}{W_{u}} \frac{d W_{u}}{d T_{s}}$ and $\frac{1}{F_{\text {net }}^{u}} \frac{d F_{\text {net }}^{u}}{d T_{s}}$ is dominated by the drastic difference between the uncoupled (AMIP) and coupled (historical and RCP4.5) experiments, between which the $T_{s}$ warming patterns are very different (Supplementary Figure 5). The correlations within each type of the experiments are not statistically significant at the $95 \%$ level partly due to the low signal-to-noise ratios for the inter-model spread relative to the noises in $\frac{1}{W_{u}} \frac{d W_{u}}{d T_{s}}$ and $\frac{1}{F_{n e t}^{u}} \frac{d F_{\text {net }}^{u}}{d T_{s}}$ within each type of model ensembles, and because many other processes besides the change of $F^{u}{ }_{n e t}$ such as the change of GMS, transient and stationary eddies and ocean heat uptake could all play a role in altering the ascent strength. Our simplified framework focuses on identifying the firstorder factors that act across all the experiments that span a wide range of surface warming patterns and model physics.

The ensemble-mean $T_{s}$ trends in the AMIP and historical runs display clear discrepancies in Eastern Pacific and Southern Oceans, and the multi-model-mean $T_{s}$ trend in the RCP4.5 runs is similar to the historical multi-model-mean (Supplementary Figure 5), suggesting that the AMIP and historical $T_{s}$ differences are mainly due to natural variability. The SST gradients can drive low-level winds and convergence, ${ }^{35}$ which directly affect evaporation and sensible heat flux. Greater lower-level convergence can promote stronger convection and higher cloud top, which can lead to stronger cloud longwave warming effect. The increase of water vapor may also contribute to net warming in the atmosphere. The enhanced energy flux into the ascending region can drive a strengthening of the ascent and induces a positive feedback between circulation and the radiation effects of clouds and water vapor, ${ }^{34,36}$ which can in turn influence the distributions of SST in the coupled simulations.

We have examined each component of $F_{\text {net }}^{u}$ in the models. It is found that the longwave cloud radiative effect at the TOA (positive for outgoing fluxes) has the highest correlation ( $R$ $=-0.52$ ) with $\frac{1}{W_{u}} \frac{d W_{u}}{d T_{s}}$ (Fig. 4b), followed by clear-sky shortwave absorption in the atmosphere $(R=0.43)$ (Fig. 4c). The models with a reduced TOA outgoing longwave radiation (OLR) associated with cloud changes tend to have a strengthening of ascent, i.e., the more longwave trapping by clouds, the greater strengthening of ascent. The increase of cloud top height, cloud amount or cloud thickness all could contribute to the reduction of OLR in the cloudy region. The discrepancies in the modeled cloud radiative effects are caused partly by different SST patterns (such as the differences between the AMIP and historical experiments) and partly by different model parameterizations of deep convection and cloud microphysics (such as the differences between the AMIP experiments). The correlation between $\frac{1}{W_{u}} \frac{d W_{u}}{d T_{s}}$ and the cloud longwave radiative effects is -0.56 in the historical experiments alone, but becomes insignificant within the AMIP and RCP4.5 experiments, indicating the effects of other processes on the ascent strength change.

Inaccurate model parameterizations of water vapor absorption of solar radiation ${ }^{37}$ and inconsistent treatment of absorbing aerosol trends ${ }^{38}$ could contribute to the clear-sky shortwave absorption disagreements (Fig. 4c). In the limited number of historical experiments that include the absorbing aerosol concentrations in the outputs, we find the changes of absorbing aerosol concentrations are positively correlated with the changes of clear-sky shortwave absorption in the ascent region (Supplementary Figure 6). Increasing absorbing aerosols would favor 
(a)

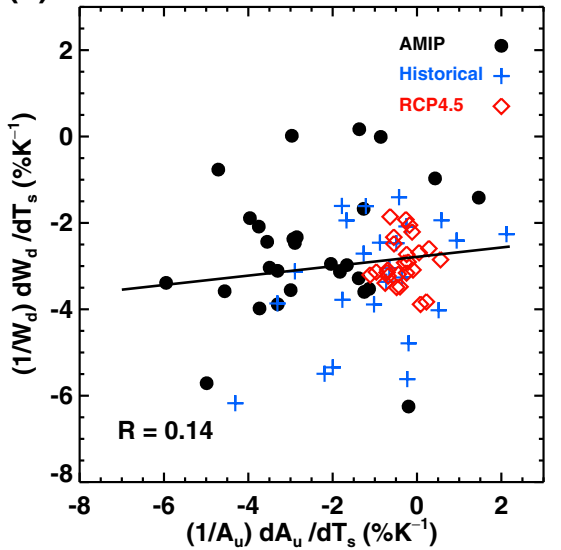

(b)

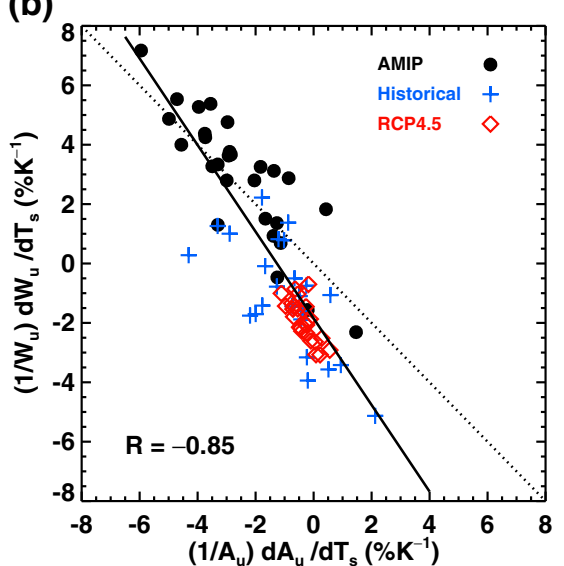

Fig. 5 Relations between the changes of descent/ascent rates and ascent area. The fractional change of a mean descent rate and $\mathbf{b}$ mean ascent rate scattered against the fractional change of the ascent area across the model simulations. All fractional changes are normalized by global-mean surface temperature trends over the same period. Each symbol represents a model experiment. The black line marks the least squares linear fit across all model experiments with correlation coefficient $(R)$ shown. The dotted line in $\mathbf{b}$ corresponds to $y=-\mathbf{x}$

stronger ascent. However, it is difficult to quantify the relative contributions of water vapor and absorbing aerosols in clear-sky shortwave absorption using the available model experiments. Nevertheless, it is clear that cloud radiative changes over the convective region add substantial diversity to the tropical ascent sensitivity to surface warming. On the contrary, the physics over the dry and non-convective region is relatively simple and consistently captured in the models with smaller inter-model spread. Albern et al. ${ }^{33}$ also found that cloud-radiative interactions amplify the model differences in the ascent response to warming in the aqua-planet simulations.

Anti-correlation between the ascent rate and area change

The mass balance between the ascending and descending boxes stipulates that the tropical ascent area change acts to offset the differential responses of $W_{d}$ and $W_{u}$ to warming (Eq. 1). The weakening of $W_{d}$ is predominantly driven by the rise of DSS, while the $W_{u}$ sensitivity to warming is largely influenced by net energy flux in the atmospheric column, for which clouds, water vapor, and aerosols can all come into play. Because the increase of DSS with warming overcomes the increase of radiative cooling in the descent region, the weakening of $W_{d}$ occurs in all model experiments irrespective of the SST warming patterns, although the magnitudes of the changes depend on the SST distributions and model physics. Constrained by the mass budget, ascent area would decrease when descent rate weakens if the ascent rate stays unchanged. When ascent rate also weakens such as in the RCP4.5 experiments or in the aqua-planet simulations under uniform SST warming, ${ }^{33}$ the ascent area can increase or decrease in order to compensate for the differential changes of descent and ascent rates. Under CMIP5 projected climate change scenarios, a tightening of ascent area is commonly simulated, ${ }^{7-9}$ suggesting there are robust physical processes at work to achieve the mass balance of tropical circulation.

Previous studies showed that the tightening of ascent area is caused by the "upped-ante" mechanism ${ }^{39}$ and the negative MSE advection $^{8}$ in a warmer climate. The "upped-ante mechanism" states that the margin of the convective zone tends to experience suppressed convection when tropospheric temperature increases because the low-level MSE required to initiate convection has increased relative to the MSE of inflow. ${ }^{39}$ The negative MSE advection argument posits that the narrowing of the ITCZ is primarily caused by the increased negative MSE advection associated with the mean Hadley Circulation acting on the strengthened MSE gradient between the tropics and subtropics. ${ }^{8}$ In addition, the MSE divergence caused by transient eddies also contributes to the narrowing of the ITCZ. ${ }^{8}$ These mechanisms are the dynamic pathways that fulfill the fundamental mass constraint.

For inter-model spread that measures the uncertainties of model simulations, the correlation between $\frac{1}{W_{d}} \frac{d W_{d}}{d T_{s}}$ and $\frac{1}{A_{u}} \frac{d A_{u}}{d T_{s}}$ across the models is only 0.14 (statistically insignificant) for all the experiments or within each type of experiments (Fig. 5a). This is because the inter-model spread in ascent area change is dominated by the large model spread in ascent rate change since the sensitivity of descent rate change per unit surface warming is similar between the models. The lack of correlation between the inter-model spreads in $\frac{1}{W_{d}} \frac{d W_{d}}{d T_{s}}$ and $\frac{1}{A_{u}} \frac{d A_{u}}{d T_{s}}$ does not violate the relations in Eq (1): we find the interannual variations of tropical ascent area are positively correlated with the variations of descent rate, with the correlations of about 0.3 to 0.5 . On the other hand, the interannual variations of $A_{u}$ and $W_{u}$ are negatively correlated with $R=-0.7$ to -0.5 for different models (figure not show).

Figure $5 \mathrm{~b}$ shows there is a remarkably high negative correlation of -0.85 between the inter-model spreads of ascent area and ascent rate sensitivity to warming across the 77 model experiments. Similar high correlations exist with $R=-0.85,-0.68$, and -0.74 for AMIP, historical and RCP4.5 experiments, respectively. This anti-correlation results from the dichotomy between the inter-model spread in the descent and ascent rate changes. The large inter-model spread in ascent rate change dominates over the small spread in the descent rate change and determines the inter-model spread of ascent area change. Although the ascent rate can weaken or strengthen depending on cloud radiative feedbacks and other net energy flux perturbations, the ascent area would respond in a way to offset the differences between the descent and ascent rate changes. In RCP4.5 and most of the historical runs, the changes of $W_{u}$ and $A_{u}$ are of the same sign. The tightening of $A_{u}$ and the weakening of $W_{u}$ work together to balance the weakened descent rate. A relative strengthening of mean ascent rate has to be counteracted by a relative tightening of the ascent area as the weakening of descent is well constrained across the models. Byrne et al. ${ }^{26}$ pointed out that such a negative correlation is expected for a given fractional change of mass transport, but they did not investigate the relationships between descent and ascent rate and area changes. Our study generalizes the tropical ascent area and strength definitions to include both 
(a)

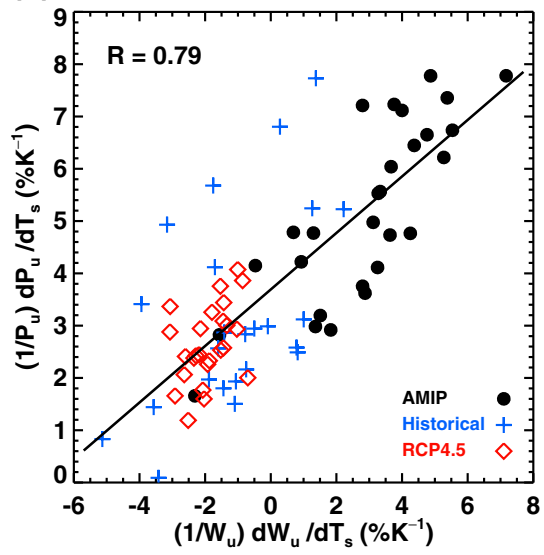

(b)

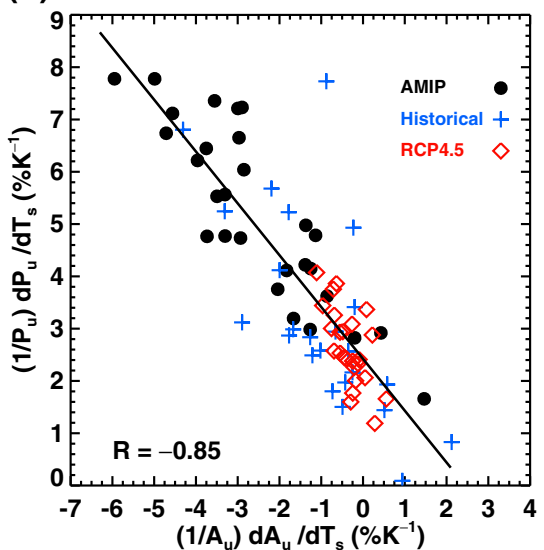

Fig. 6 The wetter the narrower precipitation change over the tropical ascending region. The fractional change of the precipitation averaged over the tropical ascending region scattered against $\mathbf{a}$ the fraction change of mean ascent rate and $\mathbf{b}$ the fractional change of the ascent area across the model simulations. All fractional changes are normalized by global-mean surface temperature trends over the same period. Each symbol represents a model experiment. The black line marks the least squares linear fit across all model experiments with correlation coefficient $(R)$ shown

the Hadley and Walker Circulation changes and employ a large number of model experiments under drastically different surface warming conditions. The mass balance and MSE budget framework allow us to quantitatively interpret the inverse relationship between the ascent area and strength changes for the tropical circulation.

More importantly, we find that cloud-radiative interactions are the leading sources of model uncertainties for ascent rate change and therefore ascent area change. It is reasonable to conjecture that the anti-correlation between $\frac{1}{A_{u}} \frac{d A_{u}}{d T_{s}}$ and $\frac{1}{W_{u}} \frac{d W_{u}}{d T_{s}}$ is sensitive to cloud radiative effects. Using the aqua-planet simulation results produced by Albern et al., ${ }^{33}$ we have computed the correlations between the ascent rate and ascent area changes for the 8 models with and without cloud-radiative interactions. It is striking that the correlation is -0.92 when cloud radiation is active but becomes -0.37 when cloud radiation is turned off (Supplementary Figure 7). Obviously, the large model spread in ascent rate change caused by the diverse cloud radiative effects is essential to the strong anti-correlation between $\frac{1}{A_{u}} \frac{d A_{u}}{d T_{s}}$ and $\frac{1}{W_{u}} \frac{d W_{u}}{d T_{s}}$.

"The wetter the narrower" ITCZ changes

The strong anti-correlation between the changes of $W_{u}$ and $A_{u}$ has close relevance to precipitation change over the ITCZ. Previous studies showed that the circulation-driven dynamic component of precipitation change dominates the model differences in regional precipitation change. ${ }^{1,2}$ Therefore, it is not surprising that the inter-model spread in mean precipitation change over the ITCZ $\left(P_{u}\right)$ is closely related with the $W_{u}$ change $(R=0.79$, Fig. $6 a) . P_{u}$ is the average rain rate over the ascending region where monthlymean $\omega_{500}<0$. Because of the strong anti-correlation between $\frac{1}{W_{u}} \frac{d W_{u}}{d T_{s}}$ and $\frac{1}{A_{u}} \frac{d A_{u}}{d T_{s}}$, the fractional change of $P_{u}$ is negatively correlated with that of $A_{u}$ with $R=-0.85$ (Fig. 6b) for all the model simulations or within each type of the experiments $(R=-0.86$, -0.66 , and -0.68 for the AMIP, historical and RCP4.5 experiments, respectively). The model simulations with a greater strengthening (or less weakening) of the ascent rate have a larger increase of precipitation over the ITCZ but the widths of the ITCZ in these models are narrower.

"The wetter the narrower" model spread in tropical rain belt change is a direct consequence of the compensating ascent rate and area changes constrained by the weakening of the descent rate in response to surface warming, a robust result across all models. Model constraints in other physical processes such as surface evaporation may also lead to "the wetter the narrower" ITCZ precipitation change as thermodynamics and dynamics in the tropics are closely coupled. The strong anti-correlation between the changes of $P_{u}$ and $A_{u}$ across the models can serve as a powerful constraint on the model simulations of regional precipitation change. There has been observational evidence for the narrowing of the meridional width of the ITCZ in the past few decades $^{40}$; however, observational metrics that represent the holistic 2-dimensional extent of the ascending region would be more appropriate for comparison with the model results presented here. Quantification of the long-term trends in the observational proxies of the ascent area is imperative to assess the model performance in capturing "the wetter the narrower" ITCZ change signal.

\section{DISCUSSIONS}

This study shows that the predominant constraint on the fractional change of the tropical ascent area is the differential responses of the descent and ascent rates to surface warming. Because the descent rate response is dominated by the increase of dry static stability with warming, a universal weakening of the descent rate is simulated in the models regardless of the warming patterns, although the magnitude of the weakening is influenced by SST distributions and convergence as wave dynamics effectively transport convection-induced tropospheric temperature anomalies throughout the tropics. ${ }^{30,41}$ Across the models, the magnitudes of descent rate change have much smaller intermodel spread than those of ascent rate change. The disparate model responses of descent and ascent rate to surface warming have important implications.

First, a weaker descent tends to be associated with a tighter ascent because of the mass balance constraint unless a greater weakening of ascent rate happens (Eq. 1). When ascent rate intensifies, a pronounced narrowing of the ascent area occurs. This argument is not limited to the Hadley Circulation. Decadal changes of the Walker Circulation could also contribute significantly to the changes of ascent area, such as in the post-1979 period. Su et al. ${ }^{9}$ showed that the tightening of the tropical ascent is correlated with a decrease of tropical-mean high altitude cloud fraction, which affects OLR and global-mean hydrological sensitivity. Thus, we infer that the tightening of ascent area can promote a macro-physical "iris" effect, ${ }^{42,43}$ in addition to the 
weakening of upper tropospheric radiative flux divergence discussed in Bony et al. ${ }^{34}$ The macro-physical "iris" effect is different from the original "iris" hypothesis that focused on the microphysical processes inside convective clouds concerning precipitation and detrainment efficiencies. ${ }^{44}$ It may not have a strong negative climate feedback as the original "iris" effect because the reduction of high cloud fraction does not necessarily lead to a net radiative cooling effect on the surface-atmosphere system. ${ }^{9}$ Byrne and Schneider $^{43}$ showed that the macro-physical "iris" effect is not likely to exert a strong influence on global climate sensitivity because the circulation-induced TOA radiative perturbations are constrained to be small on global averages and global temperature is relatively insensitive to tropical TOA perturbations.

Second, the dominance of the inter-model spread in the ascent rate change over that of the descent rate change results in a strong anti-correlation between the ascent rate and area changes across the models, leading to "the wetter the narrower" ITCZ precipitation responses. As it is possible to measure the tropical ascent area using various space-borne observational proxies, the strong correlation between the ascent area and the ITCZ precipitation enables a useful constraint on the precipitation changes.

Moreover, we show that the ascent rate change varies substantially across the models due to the model differences in simulating the radiative effects of clouds, water vapor and aerosols. Accordingly, the magnitudes of the tightening of the ascent area vary drastically between the models. To reduce the uncertainties in tropical circulation and regional precipitation projections, improving the model physics related to deep convective clouds is critically needed.

\section{METHODS}

Models

We employ 77 climate model simulations available at the CMIP5 archive (https://cmip.llnl.gov/cmip5/data_portal.html). Three types of model simulations are analyzed: 28 AMIP-type runs driven by the observed SST and sea ice from 1979 to 2005, 24 atmosphere-ocean coupled historical runs from 1979 to 2005, and 25 RCP4.5 runs from 2006 to 2100. The historical runs are driven by natural and anthropogenic forcings, universal across the models. The RCP4.5 runs project climate changes under a scenario with moderate mitigation of greenhouse gases (GHG).

All analyses are conducted over the tropical domain $\left(30^{\circ} \mathrm{S}-30^{\circ} \mathrm{N}\right)$ including both land and ocean, except for the global surface temperature trends. The annual-mean area coverage of the tropical ascent is based on the monthly mean data where $\omega_{500}<0 \mathrm{Pas}^{-1}$. The linear trends in all variables are computed using the least square linear regression.

\section{Moist static energy budget framework}

We consider the tropical circulation consisting of two boxes of horizontally uniform properties in each box: one is moist and cloudy and the other is dry and clear. Deep convection and large-scale ascent prevail in the moist box while subsidence dominates in the dry box. Combing the energy and moisture budgets together, the moist static energy (MSE) budget in the flux form ${ }^{24}$ for the moist box is expressed as

$$
\partial_{t}\langle h\rangle+\langle\nabla \cdot \mathbf{v} h\rangle+\left\langle\partial_{p} \omega h\right\rangle=F_{\text {net }},
$$

where the angle brackets $\left\langle{ }^{*}\right\rangle$ represent the mass-weighted vertical integral from the surface to the top of atmosphere (TOA) and $h$ denotes MSE, i.e., $h$ $=C_{p} T+L_{v} q+\Phi$ with $\Phi$ being geopotential. The variables $T, q, \mathbf{v}$ and $\omega$ are atmospheric temperature, moisture, horizontal winds and vertical pressure velocity, and $C_{p}$ and $L_{v}$ are specific heat at constant pressure and latent heat of condensation. The $F_{\text {net }}$ on the r.h.s. is the net energy flux into the atmospheric column. It includes the net downward radiative flux at the TOA $\left(F_{t}^{\downarrow}\right)$ and the net upward radiative flux at the surface $\left(F_{s}^{\uparrow}\right)$ plus latent $(E)$ and sensible $(H)$ heat fluxes, i.e.,

$F_{\text {net }}=F_{t}^{\downarrow}+F_{s}^{\uparrow}+E+H=\left(S_{t}^{\downarrow}-S_{t}^{\uparrow}-L_{t}^{\uparrow}\right)+\left(S_{s}^{\uparrow}-S_{s}^{\downarrow}+L_{s}^{\uparrow}-L_{s}^{\downarrow}\right)+E+H$, where $S$ and $L$ denote shortwave and longwave radiative fluxes, respectively, with the flux directions indicated by the arrows, and the subscripts $t$ and $s$ mark the TOA and surface fluxes, respectively.

Considering that tropical tropospheric temperature closely follows moist adiabats under quasi-equilibrium and the vertical velocity has a baroclinic structure with a maximum in the middle troposphere, ${ }^{24,28,29}$ we assume $\omega$ $(x, y, z, t)=-\Omega_{1}(p) W_{u}$, where $\Omega_{1}(p)$ is the vertical profile of the first baroclinic mode of the vertical pressure velocity and $W_{u}$ is the maximum strength of the vertical motion. The gross moist stability (GMS), which considers the effect of moisture on net atmospheric instability, can be defined as GMS $=\left\langle\Omega_{1}\left(-\partial_{p} h\right)\right\rangle$. Thus, the dominant control on the tropical ascent rate is $W_{u}=F_{n e t}^{u} / G M S$. For the descent box, radiative cooling balances against adiabatic warming so that $W_{d}=F_{\text {net }}^{d} / \mathrm{DSS}$. The dry static stability DSS $=-\frac{T}{\theta} \frac{\partial \theta}{\partial P}$ at $500 \mathrm{hPa}$ is used, where $T$ is temperature and $\theta$ is potential temperature. We derive DSS using monthly $T$ and $\theta$ at $500 \mathrm{hPa}$, and $\frac{\partial \theta}{\partial P}$ is computed by center differencing of $\theta$ at 400,500 , and $600 \mathrm{hPa}$. The relationships between the changes of $W_{u}, W_{d}, F_{\text {net }}^{u}$ and DSS averaged over the ascent $\left(\omega_{500}<0\right)$ and descent $\left(\omega_{500}>0\right)$ regions within $30^{\circ} \mathrm{S}-30^{\circ} \mathrm{N}$ are analyzed in the study.

\section{Significance tests for the correlation coefficients}

For 77 independent samples, the 2-sided student- $t$ test requires that the magnitude of correlation coefficient $R \geq 0.22$ for the $95 \%$ significance level and $R \geq 0.29$ for the $99 \%$ significance level. In this study, most correlation coefficients are statistically significant at the $99 \%$ significance level, unless noted otherwise.

Code availability

The analyses were conducted using MATLAB. All programing codes are available upon request from the corresponding author.

\section{DATA AVAILABILITY}

The CMIP5 model simulations used in this study are all publicly available at https:// cmip.IInl.gov/cmip5/data_portal.html. All analysis results and the code used during the study are available on request from the corresponding author.

\section{ACKNOWLEDGEMENTS}

We acknowledge the World Climate Research Programme's Working Group on Coupled Modelling, which is responsible for CMIP, and we thank the climate modeling groups for producing and making available their model output. We thank the funding support from NASA NEWS, ACMAP-AST and MAP programs. J.D.N. is supported by the NSF AGS-1540518 grant. This work was performed at Jet Propulsion Laboratory, California Institute of Technology, under contract with NASA.

\section{AUTHOR CONTRIBUTIONS}

H.S. designed the analysis and wrote the paper. C.Z., J.H.J., and L.W. analyzed the CMIP5 model simulations. J.D.N. and Y.L.Y. provided suggestions for the analysis and comments on the manuscript. Everyone edited the manuscript.

\section{ADDITIONAL INFORMATION}

Supplementary Information accompanies the paper on the npj Climate and Atmospheric Science website (https://doi.org/10.1038/s41612-019-0066-8).

Competing interests: The authors declare no competing interests.

Publisher's note: Springer Nature remains neutral with regard to jurisdictional claims in published maps and institutional affiliations.

\section{REFERENCES}

1. Bony, S. et al. Robust direct effect of carbon dioxide on tropical circulation and regional precipitation. Nat. Geosci. 6, 447-451 (2013).

2. Xie, S. P. et al. Towards predictive understanding of regional climate change. Nat. Clim. Change 5, 921-930 (2015).

3. Held, I. M. \& Soden, B. J. Robust responses of the hydrological cycle to global warming. J. Clim. 19, 5686-5699 (2006). 
4. Knutson, T. R. \& Manabe, S. Time-mean response over the tropical Pacific to increase $\mathrm{CO}_{2}$ in a coupled ocean-atmosphere model. J. Clim. 8, 2181-2199 (1995).

5. Vecchi, G. A. \& Soden, B. J. Global warming and the weakening of the tropical circulation. J. Clim. 20, 4316-4340 (2007).

6. Su, $\mathrm{H}$. et al. Weakening and strengthening structures in the Hadley Circulation change under global warming and implications for cloud response and climate sensitivity. J. Geophys. Res.: Atmospheres 119, 5787-5805 (2014).

7. Lau, W. K.-M. \& Kim, K.-M. Robust Hadley Circulation changes and increasing global dryness due to $\mathrm{CO}_{2}$ warming from CMIP5 model projections. Proc. Nat. Acad. Sci. 112, 3630-3635 (2015).

8. Byrne, M. P. \& Schneider, T. Narrowing of the ITCZ in a warming climate: Physical mechanisms. Geophys. Res. Lett. 43, 350-11,357 (2016).

9. Su, H. et al. Tightening of tropical ascent and high clouds key to precipitation change in a warmer climate, Nature. Communications 8, 15771 (2017).

10. Fu, Q., Johanson, C. M., Wallace, J. M. \& Reichler, T. Enhanced mid-latitude tropospheric warming in satellite measurements. Science 312, 1179 (2006).

11. Seidel, D. J., Fu, Q., Randel, W. J. \& Reichler, T. J. Widening of the tropical belt in a changing climate. Nat. Geosci. 1, 21-24 (2008).

12. Tanaka, H. L., Ishizaki, N. \& Kitoh, A. Trend and interannual variability of Walker, monsoon and Hadley circulations defined by velocity potential in the upper troposphere. Tellus 56A, 250-269 (2004).

13. Quan, X. W., Diaz, H. F. \& Hoerling M. P. Change in the tropical Hadley Circulation since 1950, in The Hadley Circulation: Past, Present, and Future (eds H. F. Diaz \& R. S. Bradley) pp. 85-120, (Cambridge Univ. Press, New York, 2004).

14. Mitas, C. M. \& Clement, A. Has the Hadley Circulation been strengthening in recent decades? Geophys. Res. Lett. 32, L03809 (2005).

15. Sohn, B. J. \& Park, S.-C. Strengthened tropical circulations in past three decades inferred from water vapor transport. J. Geophys. Res. 115, D15112 (2010).

16. Solomon, A. \& Newman, M. Reconciling disparate 20th century Indo-Pacific ocean temperature trends in the instrumental record. Nat. Clim. Change 2, 691-699 (2012).

17. L'Heureux, M. L., Lee, S. \& Lyon, B. Recent multidecadal strengthening of the Walker circulation across the tropical Pacific. Nat. Clim. Change 3, 571-576 (2013).

18. Sohn, B. J., Yeh, S. W., Schmetz, J. \& Song, H. J. Observational evidences of Walker circulation change over the last 30 years contrasting with GCM results. Clim. Dyn. 40, 1721-1732 (2013).

19. Vecchi, G. A., Soden, B. J., Wittenberg, A. T., Held, I. M., Leetmaa, A. \& Harrison, M. J. Weakening of tropical Pacific atmospheric circulation due to anthropogenic forcing. Nature 441, 73-76 (2006).

20. Bellomo, K. \& Clement, A. C. Evidence for weakening of the Walker circulation from cloud observations. Geophys. Res. Lett. 42, 7758-7766 (2015).

21. Meng, Q. et al. (2012) Twentieth century Walker Circulation change: data analysis and model experiments. Clim. Dyn. 38, 1757-1773 (2012)

22. Sandeep, S., Stordal, F., Sardeshmukh, P. D. \& Compo, G. P. Pacific Walker Circulation variability in coupled and uncoupled climate models. Clim. Dyn. 43, 103-117 (2014).

23. Ma, S. \& Zhou, T. Robust Strengthening and Westward Shift of the Tropical Pacific Walker Circulation during 1979-2012: A Comparison of 7 Sets of Reanalysis Data and 26 CMIP5 Models. J. Clim. 29, 3097-3118 (2016).

24. Pierrehumbert, R. T. Thermostats, radiator fins, and the run-away greenhouse. J. Atmos. Sci. 52, 1784-1806 (1995).

25. Larson, K., Hartmann, D. L. \& Klein, S. A. The role of clouds, circulation, and boundary layer structure in the sensitivity of the tropical climate. J. Clim. 12, 2359-2374 (1999).

26. Byrne, M. P., A. G. Pendergrass, A. D. Rapp, K. R. Wodzicki. Response of the intertropical convergence zone to climate change: Location, Width and strength, current climate. Change Reports, 2198-6061, https://doi.org/10.1007/s40641-0180110-5, (2018).
27. Neelin, J. D. \& Held, I. M. Modeling tropical convergence based on the moist static energy budget. Mon. Wea. Rev. 115, 3-12 (1987).

28. Chou, C. \& Neelin, J. D. Mechanisms of global warming impacts on regional tropical precipitation. J. Clim. 17, 2688-2701 (2004).

29. Su, H. \& Neelin, J. D. The scatter in tropical average precipitation anomalies. J. Clim. 16, 3966-3977 (2003).

30. Sobel, A. H., Nilsson, J. \& Polvani, L. M. The weak temperature gradient approximation and balanced tropical moisture waves. J. Atmos. Sci. 58, 3650-3665 (2001).

31. Neelin, J. D. \& Zeng, N. A quasi-equilibrium tropical circulation model-Formulation. J. Atmos. Sci. 57, 1741-1766 (2000).

32. Wills, R. C., Levine, X. J. \& Schneider, T. Local energetic constraints on Walker circulation strength. J. Atmos. Sci., 2017. https://doi.org/10.1175/JAS-D-16-0219.1. (2017). ISSN 0022-4928.

33. Albern, N., Voigt, A., Buehler, S. A., \& Grützun, V. Robust and nonrobust impacts of atmospheric cloud-radiative interactions on the tropical circulation and its response to surface warming. Geophysical Research Letters, 45, https://doi.org/ 10.1029/2018GL079599 (2018).

34. Bony., S. et al. Thermodynamic control of anvil cloud amount. Proc. Nat. Acad. Sci. 113, 8927-8932 (2016).

35. Lindzen, R. S. \& Nigam, S. On the role of sea surface temperature gradients in forcing low-level winds and convergence in the tropics. J. Atmos. Sci. 44, 2418-2436 (1987)

36. Voigt, A., and T. A. Shaw, Circulation response to warming shaped by shaped by radiative changes of clouds and water vapour. Nature Geoscience, 8, https://doi. org/10.1038/NGEO2345 (2015).

37. DeAngelis, A. M., Qu, X., Zelinka, M. D. \& Hall, A. An observational radiative constraint on hydrologic cycle intensification. Nature 528, 249-253 (2015).

38. Pendergrass, A. G. \& Hartmann, D. L. Global-mean precipitation and black carbon in AR4 simulations. Geophys. Res. Lett. 39, L01703 (2012).

39. Neelin, J. D., Chou, C. \& Su, H. Tropical drought regions in global warming and El Nino teleconnections. Geophys. Res. Lett. 30, 2275 (2003).

40. Wodzicki, K. R. \& Rapp, A. D. Long-term characterization of the Pacific ITCZ using TRMM, GPCP, and ERA-Interim. J. Geophys. Res. Atmos. 121, 3153-3170 (2016).

41. Su, H., Neelin, J. D. \& Meyerson, J. E. Sensitivity of tropical tropospheric temperature to sea surface temperature forcing. J. Clim. 16, 1283-1301 (2003).

42. Choi, Y. et al. Revisiting the iris effect of tropical cirrus clouds with trmm and atrain satellite data. J. Geophys. Res.: Atmospheres 122, 5917-5931 (2017).

43. Byrne, M. P. \& Schneider, T. Atmospheric dynamics feedback: concept, simulations and climate implications. J. Clim. 31, 3249-3264 (2018).

44. Lindzen, R. S., Chou, M. -D. \& Hou, A. U. Does the Earth have an adaptive infrared iris? Bull. Am. Meteorol. Soc. 82, 417-432 (2001).

(i) Open Access This article is licensed under a Creative Commons Attribution 4.0 International License, which permits use, sharing, adaptation, distribution and reproduction in any medium or format, as long as you give appropriate credit to the original author(s) and the source, provide a link to the Creative Commons license, and indicate if changes were made. The images or other third party material in this article are included in the article's Creative Commons license, unless indicated otherwise in a credit line to the material. If material is not included in the article's Creative Commons license and your intended use is not permitted by statutory regulation or exceeds the permitted use, you will need to obtain permission directly from the copyright holder. To view a copy of this license, visit http://creativecommons. org/licenses/by/4.0/.

(c) The Author(s) 2019 